\title{
Accuracy of biomarkers obtained from cone beam computed tomography in assessing the internal trabecular structure of the mandibular condyle
}

\author{
Fouad H. Ebrahim, DDS, MS, ${ }^{\text {a }}$ Antonio C.O. Ruellas, DDS, MS, PhD, ${ }^{\text {b,c }}$ Beatriz Paniagua, PhD, \\ Erika Benavides, DDS, MS, PhD, ${ }^{\mathrm{a}}$ Karl Jepsen, MS, PhD,${ }^{\mathrm{e}}$ Larry Wolford, DMD, ${ }^{\mathrm{f}}$ \\ Joao Roberto Goncalves, DDS, MS, PhD, ${ }^{g}$ and Lucia H. S. Cevidanes, DDS, MS, PhD ${ }^{a}$
}

\begin{abstract}
Objective. The aim of this study was to validate the ability of cone beam computed tomography (CBCT) to measure condylar internal trabecular bone structure and bone texture parameters accurately.

Study Design. Sixteen resected condyles of individuals undergoing temporomandibular joint replacement were collected and used as samples. These condyles were then radiographically imaged by using clinically oriented dental CBCT and research oriented micro-computed tomography (micro-CT). The CBCT scans were then compared with the gold standard micro-CT scans in terms of 21 bone imaging parameters. Descriptive histologic investigation of the specimens was also performed.

Results. Significant correlations were found for several imaging parameters between the CBCT and micro-CT images, including trabecular thickness $(r=0.92)$, trabecular separation $(r=0.78)$, bone volume $(r=0.90)$, bone surface area $(r=0.79)$, and degree of anisotropy measurements $(r=0.77)$.

Conclusions. Measurements of trabecular thickness, trabecular separation, bone volume, bone surface area, and degree of anisotropy obtained from high-resolution dental CBCT images may be suitable bone imaging biomarkers that can be utilized clinically and in future research. (Oral Surg Oral Med Oral Pathol Oral Radiol 2017;124:588-599)
\end{abstract}

The temporomandibular joint (TMJ) is one of the most complex joints in the human body and allows for both hingelike rotational and translational movements of the mandible. ${ }^{1}$ Similar to other diarthodial (freely moving) joints of the body, the TMJ can also be affected by osteoarthritis (OA). TMJ OA is a degenerative disease characterized by progressive degradation of articular cartilage and a simultaneous remodeling of the underlying bone of the mandibular condyle and glenoid fossa. ${ }^{2}$ These degenerative changes that lead to structural alterations in TMJ joint morphology can also result in severe pain, loss of function, altered occlusion, difficulty opening, asymmetry, crepitus, clicking, anterior open bite, and backward rotation of the mandible. ${ }^{3,4}$

Research reported in this publication was supported by the National Institute of Dental \& Craniofacial Research of the National Institutes of Health (Award Number R01 DE024450). The content is solely the responsibility of the authors and does not necessarily represent the official views of the National Institutes of Health.

${ }^{a}$ School of Dentistry, University of Michigan, Ann Arbor, MI, USA.

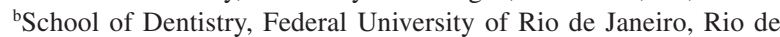
Janeiro, RJ, Brazil.

${ }^{\mathrm{c} D e p a r t m e n t}$ of Pediatric Dentistry and Orthodontics, Visiting Postdoctoral Scholar, School of Dentistry, University of Michigan, Ann Arbor, MI, USA.

${ }^{\mathrm{d}}$ University of North Carolina, Chapel Hill, NC, USA.

${ }^{\mathrm{e} B i o m e d i c a l ~ S c i e n c e s ~ R e s e a r c h ~ B u i l d i n g, ~ U n i v e r s i t y ~ o f ~ M i c h i g a n, ~ A n n ~}$ Arbor, MI, USA.

${ }^{\mathrm{f} B a y l o r ~ U n i v e r s i t y ~ M e d i c a l ~ C e n t e r, ~ D a l l a s, ~ T X, ~ U S A . ~}$

${ }^{\mathrm{g}}$ Araraquara Dental School, Paulista State University, Araraquara, SP, Brazil.

Received for publication Mar 23, 2017; returned for revision Aug 3, 2017; accepted for publication Aug 12, 2017.

(c) 2017 Elsevier Inc. All rights reserved.

2212-4403/\$ - see front matter

https://doi.org/10.1016/j.oooo.2017.08.013
The prevalence rates of TMJ OA vary across different studies because of differing diagnostic criteria, but radiographic evidence of TMJ degeneration is seen in $8 \%$ to $16 \%$ of the population. ${ }^{5}$ TMJ OA can affect one or both TMJs and shows a strong predilection for middleaged women. ${ }^{6}$ TMJ OA is a slowly progressing disease that can take years to appear clinically, and most manifestations of TMJ OA follow a natural course independent of treatment, reaching a stable, nonpainful burnout endpoint phase after a period of active joint disease. ${ }^{7}$ However, a small group of patients $(<20 \%)$ undergo rapid and widespread degenerative changes, chronic pain, occlusal changes, and impaired quality of life and require surgical intervention. ${ }^{8}$ Unfortunately, there is no way to predict the course or severity of the disease, and no single clinical finding specific to TMJ OA exists. ${ }^{7}$

The initial morphologic changes in TMJ OA are subtle and subclinical, with or without significant symptoms, and therefore many patients may not present symptoms in the early stages. Unfortunately, after such joint changes have occurred and TMJ OA reaches a point where it is clinically and radiographically detectable, it is not possible to restore the condyle to its original morphology. ${ }^{9}$

Medical computed tomography (CT) is currently the gold standard for imaging degenerative osseous changes

\section{Statement of Clinical Relevance}

The analysis of the internal trabecular architecture of the resected condylar heads may be a method that helps diagnose temporomandibular joint osteoarthritis early without having to wait until surface changes of cortical bone become visible. 
associated with TMJ OA and has shown an $84 \%$ accuracy in detecting these arthritic changes. ${ }^{10}$ However, medical CT has many drawbacks, such as size and cost of the machine, cost per scan, high radiation dose per scan, and unfamiliarity to dentists who are often the first point of contact for patients with TMJ disorders.

Recently, CBCT has emerged as the radiographic modality of choice for imaging the osseous components of the TMJ because it has been shown to be as effective as conventional medical $\mathrm{CT}$ in detecting bony changes in the joint. ${ }^{11,12}$

Several studies have observed specific architectural changes in the subchondral bone of other joints that have been also affected by OA, such as the knee, hip, or vertebrae. ${ }^{13-15}$ These findings support the notion that changes in the subchondral bone of the TMJ may be indicative of an underlying disease process and thus could possibly be used for early diagnosis. Therefore, analysis of the internal trabecular architecture of the TMJ may potentially be a method to diagnose the disease early without having to wait until frank evidence of surface changes of cortical bone becomes visible much later in the disease process.

The literature contains several reports of studies on structural bone parameters to assess the internal structure of trabecular bone, such as trabecular thickness, trabecular separation, bone volume, bone volume fraction, bone surface area, degree of anisotropy, structural model index, ellipsoid factor, connectivity, connectivity density, and the Euler characteristic. ${ }^{16,17}$ Furthermore, another technique that has been commonly used to compare images in biomedical imaging literature is gray-level run length. A gray-level run is a set of consecutive, collinear picture points having the same gray-level value. The length of a certain run corresponds to the number of collinear picture points. The computation of gray-level run-length features is based on the gray-level run-length matrix, which describes each pixel's neighborhood local texture. Different gray level runlength features correspond to different values for homogeneous, heterogeneous, coarse, or fine textures. ${ }^{18}$ To date, no studies have attempted to compare bone texture in CBCT and micro-CT images using gray level run-length analysis. The aim of this study was to validate the ability of CBCT to measure condylar internal trabecular bone structure and bone texture parameters accurately.

\section{MATERIALS AND METHODS}

The protocol for the CBCT assessment of the internal trabecular structure of the mandibular condyle was reviewed by the University of Michigan institutional review board and was deemed to have "not regulated" status and thus did not require approval.

\section{Sample}

A sample of 16 resected condyles was collected from 14 patients undergoing total joint replacement of either one or both TMJs. Condylar resections were performed by one operator, an oral and maxillofacial surgeon, in Dallas, Texas. A clinical examination, based on the Diagnostic Criteria for Temporomandibular Disorders (DC/TMD), ${ }^{19}$ was performed on all patients by a clinician trained and experienced in DC/TMD examination and diagnoses. All cases met the DC/TMD axis I group III criteria for TMJ $\mathrm{OA},{ }^{19}$ and the radiographic diagnosis criteria ${ }^{20}$ were assessed with CBCT performed for diagnostic purposes.

All condyles in the sample were from females ranging in age from 18 to 64 years. The review of the patients' medical history excluded any patients with systemic or metabolic conditions. The samples were fixed in $10 \%$ neutral buffered formalin and then stored in $70 \%$ ethanol at freezing temperatures until analysis was performed.

\section{Three-dimensional radiographic image acquisition}

CBCT scans of each resected condyle were obtained at the University of Michigan School of Dentistry, using a three-dimensional (3-D) Accuitomo 170 (J Morita Mfg Corp., Tokyo, Japan) with a localized $40 \times 40 \mathrm{~mm}$, small field of view. All samples were carefully oriented in a positioning cup to standardize and calibrate their placement within the acquired CBCT images. The primary reconstruction of the acquired volumetric images was performed in isotropic $80 \mu \mathrm{m}$ voxels with 500 axial slices. Exposures were made using an 18 -second scan time at $90 \mathrm{kVp}$ and $1 \mathrm{~mA}$.

Micro-CT scans of each resected condyle were also obtained at the University of Michigan School of Dentistry, using a $\mu$ CT 100 unit (Sanco Medical, Bssersdorf, Switzerland). Specimens were embedded in $1 \%$ agarose before scan acquisition. Scans were acquired at a medium resolution setting of $40 \mu \mathrm{m}$ voxel size with an integration time of $500 \mathrm{~ms}$ at $90 \mathrm{kVp}$ and $155 \mu \mathrm{A}$.

\section{3-D radiographic image analysis}

The image analysis was performed in 6 steps as described below.

Step 1: 3-D reconstructions of the CBCT and micro-CT scans. Reconstructions of the 3-D volumetric label maps from the CBCT and micro-CT scans were performed using ITK-SNAP v. 2.4 (open-source software, www.itksnap.org). First, DICOM (Digital Imaging and Communications in Medicine) files from both the CBCT and micro-CT scans were imported into ITK-SNAP. Trabecular bone was segmented and labeled using a semiautomated, one-sided thresholding procedure in which voxels with gray values above a given value were labeled. For the CBCT images, this lower threshold value was initially set to a gray-scale value of 1400 and then manually adjusted to best visualize the trabeculae. Lower threshold values were maintained within a range of 1200 to 1600 to ensure consistency. Because of the different intensity of the micro-CT scans, the lower threshold value 


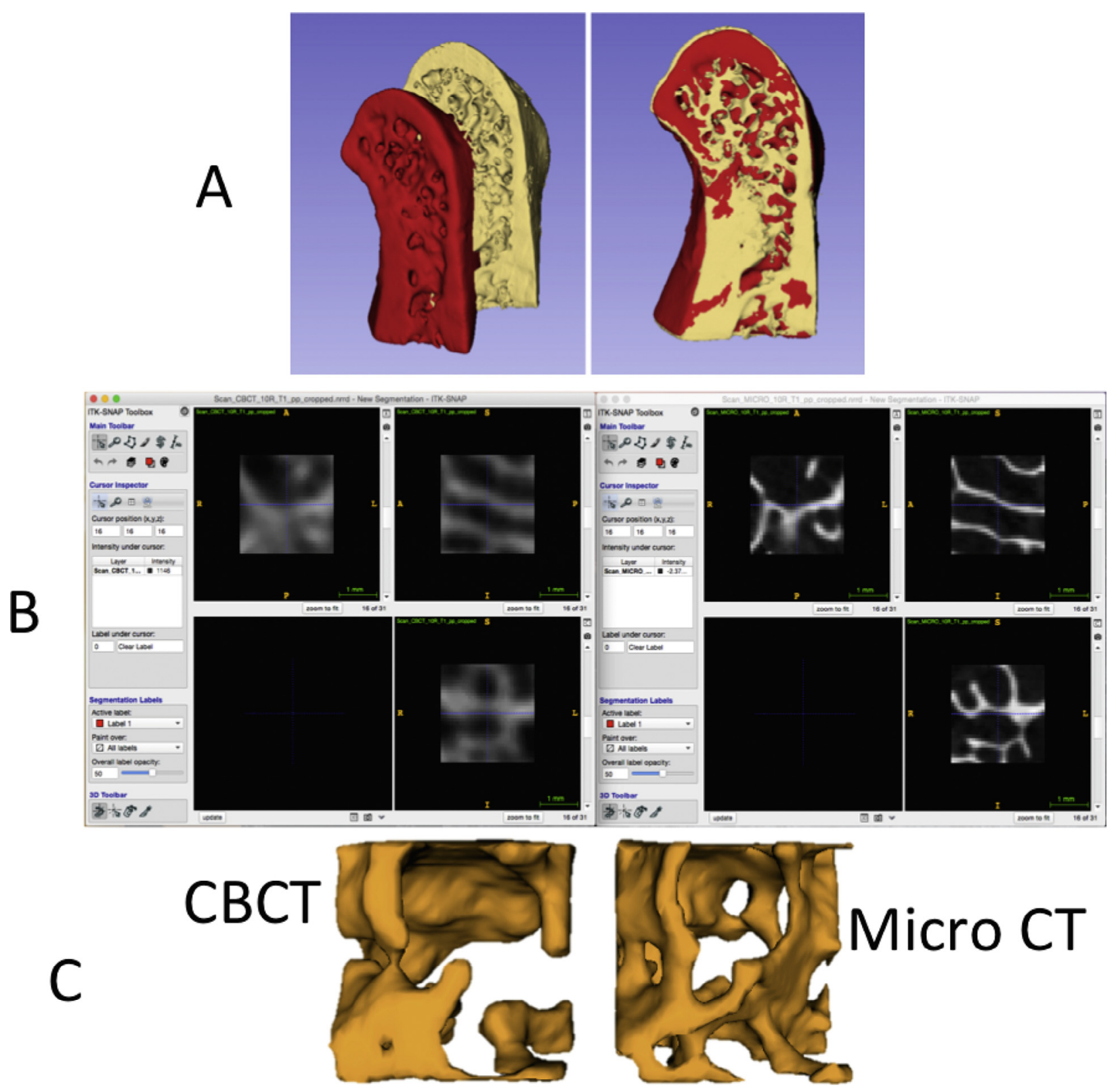

Fig. 1. A, Three-dimensional (3-D) surface models of the condyles from both the cone beam computed tomography (CBCT) (red) and micro-CT (beige) scans were approximated and registered in Slicer software. B, A representative trabecular region of interest for both CBCT (left) and micro-CT (right) images. Region of interest crop boxes measured $2.48 \mathrm{~mm}^{3}$ for both imaging modalities and were registered on the same coordinate plane. C, Cropped CBCT and micro-CT 3-D volume models generated from regions of subchondral bone defects.

of these scans was initially set to 21,000 and adjusted within a range of 19,000 to 23,000 to best visualize the trabeculae. The final 3-D volumetric label maps were converted and then saved as .stl files using the "Model Maker" tool in 3-D Slicer (open-source software, www.slicer.org).

Step 2: Registration of the micro-CT to CBCT scans. As the orientations of the CBCT and micro-CT scans were not the same, there was a need to reorient and register the 3-D surface models such that both the CBCT and micro-CT scans could be compared with each another. The 3-D surface models were opened in 3-D Slicer, and 3-D models of each condyle for both CBCT (red) and micro-CT (beige) scans were approximated and registered (Figure 1A).

A 2-step registration process was employed to register the corresponding micro-CT and CBCT 3-D surface models for each condyle. First, using the "Transform" tool within 3-D Slicer, the micro-CT 3-D surface model for each condyle was manipulated and approximated to the corresponding CBCT 3-D volume model, which 
Table I. Comparison of gray-level run-length bone texture parameters from the cropped cone beam computed tomography (CBCT) and micro-CT volumes of the defective regions

\begin{tabular}{|c|c|c|c|c|}
\hline $\begin{array}{l}\text { Grey level run-length parameters } \\
\text { (cropped scans) }\end{array}$ & Correlation & $\begin{array}{c}C B C T \\
(\text { mean } \pm S D)\end{array}$ & $\begin{array}{c}\text { Micro-CT } \\
(\text { mean } \pm S D)\end{array}$ & Difference \\
\hline Short run emphasis & -0.14 & $0.76 \pm 0.09$ & $0.95 \pm 0.05$ & $-0.19 *$ \\
\hline Long run emphasis & -0.33 & $262 \pm 145$ & $27 \pm 43$ & $235^{*}$ \\
\hline Gray-level nonuniformity & -0.05 & $401 \pm 67$ & $486 \pm 79$ & $-86^{\dagger}$ \\
\hline Run-length nonuniformity & -0.43 & $7666 \pm 1333$ & $12,535 \pm 2229$ & $-4869^{*}$ \\
\hline Low gray-level run emphasis & 0.03 & $0.08 \pm 0.02$ & $0.10 \pm 0.02$ & $-0.02^{\dagger}$ \\
\hline High gray-level run emphasis & $0.73 *$ & $1257 \pm 291$ & $1466 \pm 333$ & $-209^{*}$ \\
\hline Short run low gray-level emphasis & 0.04 & $0.05 \pm 0.02$ & $0.09 \pm 0.02$ & $-0.03 *$ \\
\hline Short run high gray-level emphasis & $0.61^{\dagger}$ & $966 \pm 217$ & $1372 \pm 246$ & $-406^{*}$ \\
\hline Long run low grey level emphasis & -0.05 & $33.53 \pm 21.03$ & $9.37 \pm 7.55$ & $24.16^{*}$ \\
\hline Long run high grey level emphasis & -0.18 & $330,462 \pm 223,708$ & $57,458 \pm 138,340$ & $273,003 *$ \\
\hline
\end{tabular}

$S D$, standard deviation.

$* P<.001$.

${ }^{\dagger} P<.01$.

served as a template. This approximation generated a transformation matrix that contained data quantifying the rotations and translations used to reach the manual approximation. The transformed 3-D surface model of the micro-CT was saved.

Second, the approximated CBCT and micro-CT 3-D surface models of each condyle were then registered to one another by performing a surface registration using the "CMF Registration" tool in 3-D Slicer. This tool automatically manipulated the micro-CT model to achieve the best fit on the CBCT model. Similarly, this step also generated a matrix that quantified the subsequent transformations needed for the registration. The registered 3-D surface model of the micro-CT was saved.

Last, the 2 transformation matrices for each condylethe approximation matrix and the registration matrix - were applied to the original micro-CT scans to reorient and register them to the corresponding CBCT scan. Then, both the micro-CT scans and 3-D volume models were reformatted to the same voxel size as the CBCT in 3-D Slicer. The goal of normalizing the voxel size between CBCT and micro-CT was to test whether bone texture is detected similarly in both imaging modalities at the smaller voxel size currently possible for TMJ imaging protocol of patients.

Step 3: Identification of subchondral bone defects in $C B C T$ and micro-CT scans. To identify regions of interest of subchondral bone defects, the registered CBCT and micro-CT scans for each condylar specimen were opened side by side and scrolled through slice by slice. A subchondral defect was recorded as an area in the bone beneath the cortical surface that appeared as a cyst and where the regular trabecular architecture was interrupted. Subchondral cysts are described as radiographic criterion of TMD.$^{19}$ Ahmad et al. ${ }^{20}$ explained that the term subcortical or subchondral cyst is, in fact, a misnomer because it is not a true cyst but, rather, a region of osseous degeneration. The "xyz" coordinates at the center of each region of interest were recorded.

Step 4: Trabecular image cropping. At each region of interest, the CBCT and micro-CT scans and 3-D volume models were cropped by 15 slices in each direction from the recorded "xyz" coordinates. Thus, for each region of interest, square crop boxes of each volume measuring $31 \times 31 \times 31$ slices or $2.48 \times 2.48 \times 2.48 \mathrm{~mm}$ were generated (Figure 1B and 1C).

Step 5: Quantification of trabecular architecture. Quantification of trabecular architecture was done using ImageJ software (open-source software, https:// imagej.nih.gov/ij/index.html). The CBCT and microCT 3-D volume crop boxes of each region of interest were opened in ITK-SNAP and exported as MetaImage files (.mha), which could then be imported into ImageJ. The 3-D crop boxes were then analyzed using the BoneJ plugin (open-source software, www.bonej.org) for trabecular thickness, trabecular separation, bone volume, bone volume/total volume, bone surface area, degree of anisotropy, structural model index, and ellipsoid factor. The 3-D crop boxes were then processed using the "Purify" tool in BoneJ to condense the trabecular network into a single, interconnected element that could be used as an input into the connectivity, connectivity density, and Euler characteristic analyses. BoneJ has been validated as an accurate tool to measure many of these bone structure parameters. ${ }^{21}$

Step 6: Bone texture analysis. Gray-level run-length coding was performed on the crop boxes of the CBCT and micro-CT scans, and the 10 gray-level run-length bone texture parameters (Table I) were analyzed using Linux scripts.

\section{Histology}

After radiographic image acquisition, the histologic evaluation was performed in 3 steps: The condyle specimens 


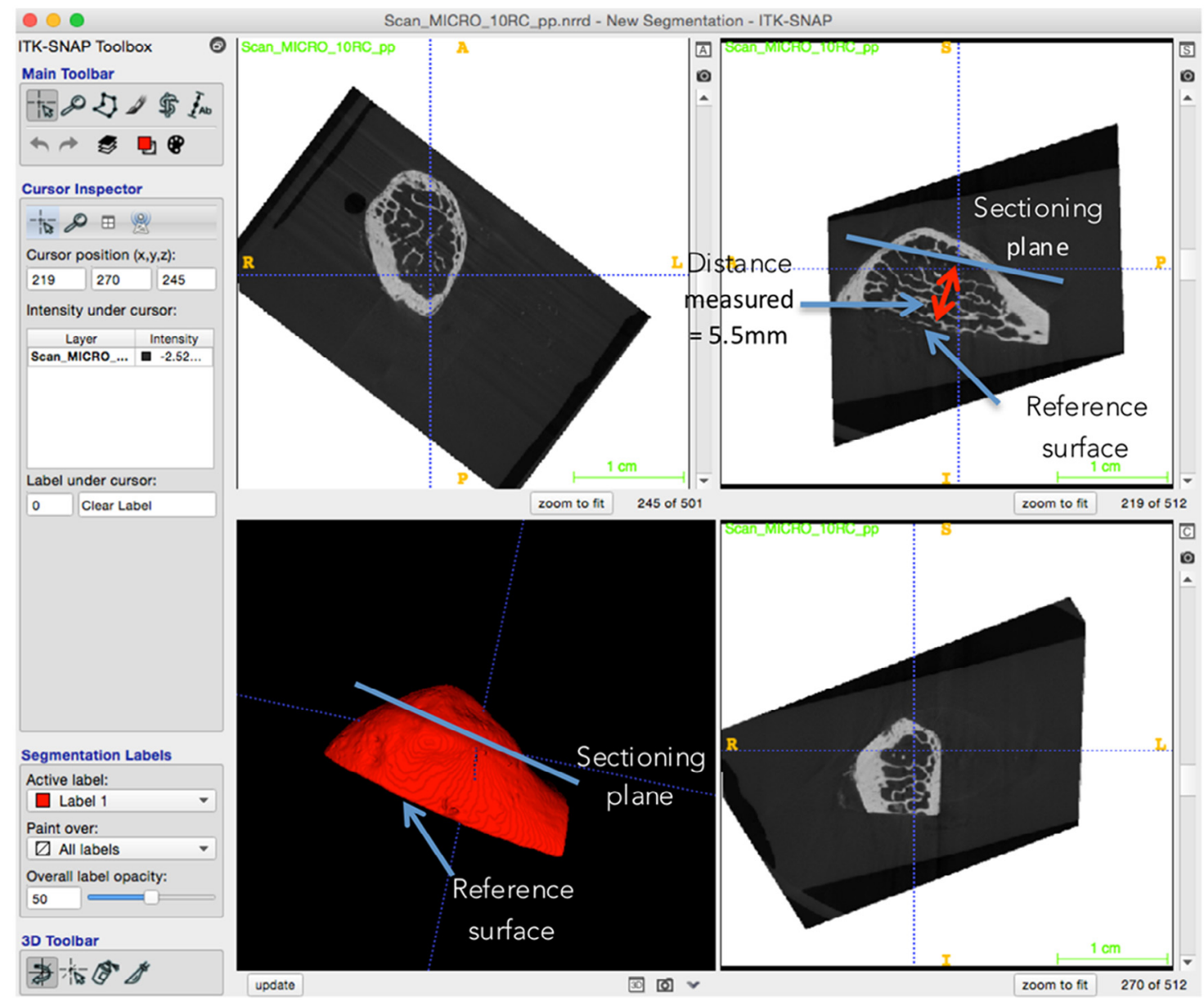

Fig. 2. Schematic picture showing the methodology that was used to determine the sectioning planes for histology and the depth of the slices. A reference plane was chosen in relation to a readily identifiable surface, the distance from the defect was measured perpendicular to this plane and the sectioning cut was then made parallel to the reference plane at the appropriate depth.

were sectioned, stained, and analyzed using light microscopy to assess the localization of osteoclasts on the defective trabecular regions.

Step 1: Sectioning. The condyles were decalcified with $10 \%$ ethylenediaminetetraacetic acid for 6 weeks. After decalcification, specimens were dehydrated using a coldgraded ethanol series. The 3-D surface models of each specimen were used to carefully identify the direction and location for sectioning, marking, and annotating how the histology sectioning should be performed to match the scans' slices as closely as possible. One previously identified subchondral defect was picked per specimen as the region of interest to prepare. To ensure that the histologic sections transected the chosen subchondral defect, the distance of the defect from an easily identifiable surface (reference surface) was measured radiographically by counting how many slices the defect was from the surface and then multiplying this by the voxel size (Figure 2). For instance, if the defect was 50 slices from the reference surface and the voxel size was $80 \mu \mathrm{m}$, then the defect was $4 \mathrm{~mm}$ from the reference surface. Specimens were first embedded in paraffin wax. Sectioned cuts of 6- $\mu \mathrm{m}$ thickness were then made parallel to the reference surface at a depth corresponding to the depth of the regions of interest.

Step 2: Staining. The biologic tissues were then stained with tartrate-resistant acid phosphatase (TRAP) to visualize osteoclasts. ${ }^{22}$ Sections were washed with $0.1 \mathrm{M}$ acetate buffer ( $\mathrm{pH}$ 5.0). They were then incubated with naphthol AS-MX phosphate and red violet LB salt (Sigma, St. Louis, MO), which was diluted in $0.1 \mathrm{M}$ acetate buffer (pH 5.0) containing $50 \mathrm{mM} / \mathrm{L}$ tartaric acid. Sections were incubated at $37^{\circ} \mathrm{C}$ for 30 minutes. Sections were then counterstained for hematoxylin (WAKO, Osaka, Japan) and mounted on slides.

Other sections were also stained with hematoxylin and eosin, which is the gold standard stain to visualize bone. ${ }^{22}$ Sections were then mounted on slides.

Step 3: Microscopy and histomorphometry. Histomorphometric analysis was done using Nikon NIS Elements Advanced software (Nikon Instruments Inc., Tokyo, Japan). The defect of interest was identified, and these 
Table II. Comparison of trabecular bone structure parameters from the cropped cone beam computed tomography (CBCT) and micro-CT volumes of the defective regions

\begin{tabular}{lcccc}
\hline $\begin{array}{l}\text { Bone Structure Parameters } \\
\text { (Cropped 3-D volumes }\end{array}$ & Correlation & $\begin{array}{c}\text { CBCT } \\
(\text { mean } \pm S D)\end{array}$ & $\begin{array}{c}\text { Micro-CT } \\
(\text { mean } \pm \text { SD) }\end{array}$ & Difference \\
\hline Trabecular thickness mean $(\mathrm{mm})$ & $0.92^{*}$ & $1.12 \pm 0.55$ & $0.67 \pm 0.34$ & $0.44^{*}$ \\
Trabecular separation mean $(\mathrm{mm})$ & $0.78^{*}$ & $1.04 \pm 0.36$ & $0.84 \pm 0.30$ & $0.21^{*}$ \\
Bone volume $\left(\mathrm{mm}^{3}\right)$ & $0.90^{*}$ & $7.78 \pm 3.07$ & $6.69 \pm 2.61$ & $1.09^{\dagger}$ \\
Bone volume/total volume & $0.90^{*}$ & $0.51 \pm 0.20$ & $0.44 \pm 0.17$ & $0.07^{\dagger}$ \\
Bone surface area $\left(\mathrm{mm}^{2}\right)$ & $0.79^{*}$ & $25.78 \pm 8.86$ & $21.21 \pm 10.21$ & $4.57^{\dagger}$ \\
Structural model index & $0.60^{\dagger}$ & $3.34 \pm 0.62$ & $3.63 \pm 1.22$ & -0.29 \\
Degree of anisotropy & $0.77^{*}$ & $0.76 \pm 0.19$ & $0.73 \pm 0.15$ & 0.03 \\
Euler characteristic & 0.35 & $-3.30 \pm 3.08$ & $-23.25 \pm 19.32$ & $19.95^{*}$ \\
Connectivity & $0.47^{+}$ & $5.67 \pm 3.75$ & $26.19 \pm 19.29$ & $-20.52^{*}$ \\
Connectivity density $\left(/ \mathrm{mm}^{3}\right)$ & $0.47^{*}$ & $0.37 \pm 0.25$ & $1.72 \pm 1.26$ & $-1.35^{*}$ \\
Ellipsoid factor $(\%)$ & 0.28 & $11.92 \pm 6.53$ & $12.25 \pm 4.48$ & -0.33 \\
\hline
\end{tabular}

$S D$, Standard deviation.

$* P<.001$

${ }^{\dagger} P<.01$.

$P<.05$

specific areas were photographed at $\times 100$ to visualize any TRAP-positive cells. Five sequential slices for each sample were photographed at the defect location. Osteoclasts were defined as TRAP-positive multinucleated cells on the bone surface or in resorptive lacunae. Trabecular bone perimeter was also calculated using the measure distance tool in NIS-Elements by manually tracing the bone perimeter in each of the slides. Osteoclasts were counted by 2 independent observers for 5 sequential slides. The counts were averaged between the 2 observers.

\section{Statistical analysis}

For CBCT and micro-CT volumes of each region of interest, 10 gray-level run-length bone texture parameters (Table I) and 11 trabecular bone structure parameters (Table II) were computed. Paired $t$ tests were chosen to evaluate if there was a consistent difference in the mean values of the 21 imaging parameters as measured on the CBCT and microCT scans. Pearson correlation analysis was chosen to assess if the same measured variable was linearly correlated between the 2 different imaging modalities.

Similarly, a Pearson correlation analysis was also carried out to determine if the osteoclast number and density from the histomorphometric analysis were linearly correlated with any of the 21 imaging parameters calculated from the CBCT and micro-CT images.

\section{RESULTS}

\section{Comparison of trabecular bone structure parameters between the CBCT and micro-CT volumes}

The 11 trabecular bone structure parameters that were computed from the cropped CBCT and micro-CT 3-D volumes of the defective trabeculae regions were first compared using paired $t$ tests, which showed very similar findings (Table II). Measurements of trabecular thickness, trabecular separa- tion, bone volume, and bone surface area were significantly greater on the CBCT images than on the micro-CT images. The measurements of the Euler characteristic, connectivity, and connectivity density were significantly lower on the CBCT images than on the micro-CT images. Last, no significant difference was observed for the structural model index, degree of anisotropy, or ellipsoid factor.

The Pearson correlation coefficients showed similar trends between the trabecular bone structure parameters measured on the cropped CBCT and micro-CT volumes (Table II; Figure 3$)$. Strong correlations $(P<.01)$ of greater than $\mathrm{r}=0.6$ were observed for 7 of the 11 trabecular bone structure parameters.

\section{Comparison of bone texture parameters between the CBCT and micro-CT volumes}

The paired $t$ tests between the 10 gray-level run-length bone texture parameters measured on the cropped CBCT and micro-CT scans showed a significant difference in all bone texture measurements (Table I). Strong Pearson correlation coefficients were observed for high graylevel run emphasis and short-run high gray-level emphasis parameters $(P<.01)$.

\section{Histomorphometry of osteoclast number and density}

A summary of the bone perimeter, number of osteoclasts, and osteoclast density of each sectioned specimen can be found in Table III. Although osteoclasts were not found in all specimens, it appeared that the osteoclasts were increased in regions close to the subchondral defects in most specimens. Pearson correlations between osteoclast number and density and the 21 imaging variables from the $\mathrm{CBCT}$ and micro-CT volumes for the corresponding specimens were calculated (Figure 4).

For CBCT (Table IV), mild positive correlations were observed between osteoclast number $(0.42)$ and 

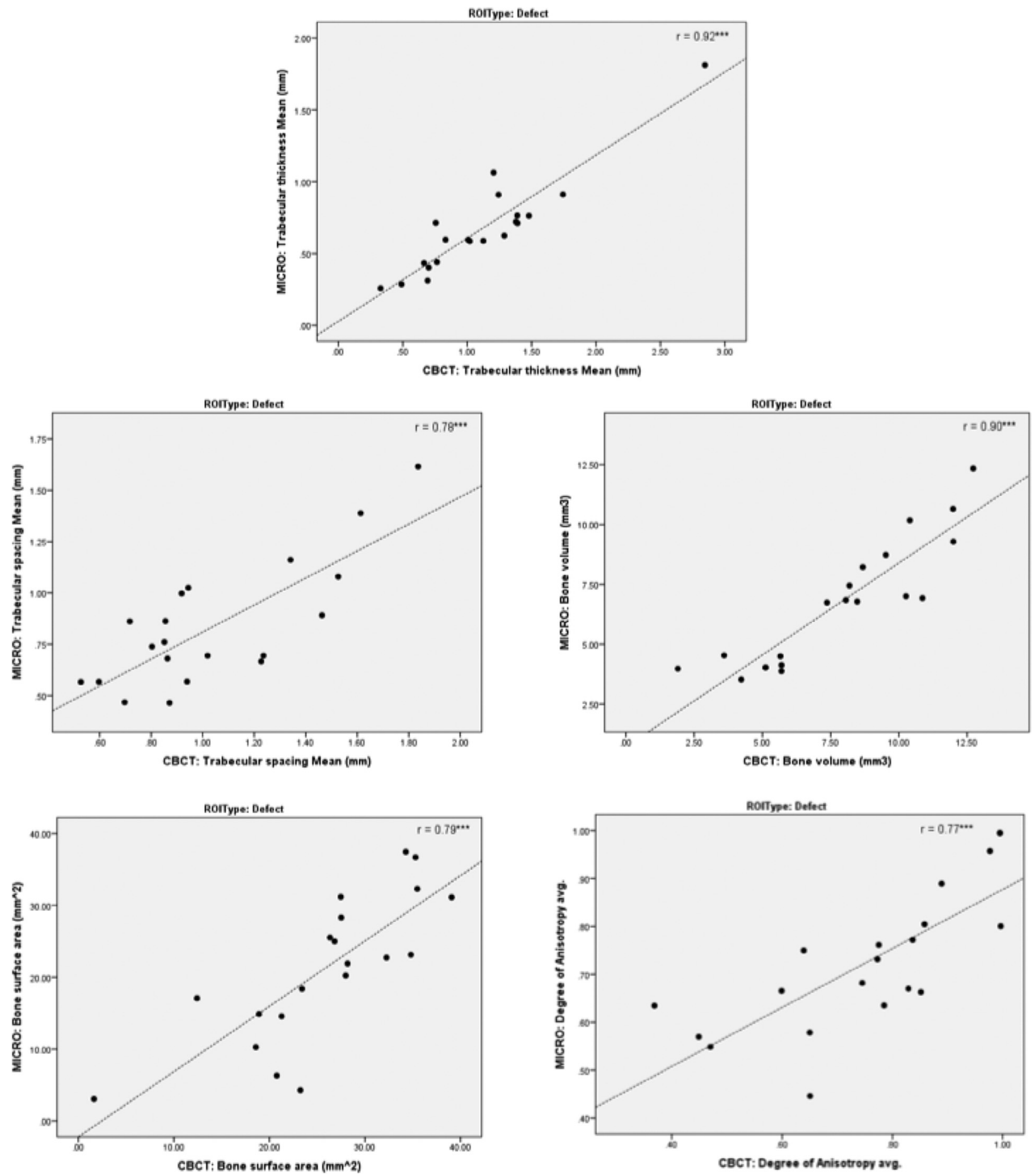

Fig. 3. Scatterplots showing correlations and linear regression between the trabecular bone structure parameters measured on the cropped cone beam computed tomography $(\mathrm{CBCT})$ and micro-CT volumes. Only statistically significant associations are illustrated. Insignificant associations were omitted. Note: $* P<.05, * * P<.01, * * * P<.001$.

osteoclast density (0.48) with ellipsoid factor. Mild negative correlations were also observed between osteoclast number and low gray-level run emphasis $(-0.46)$ and longrun low gray-level emphasis $(-0.44)$.

For micro-CT (Table IV), moderate positive correlations were found between osteoclast number and several trabecular bone structure and bone texture measurement, including connectivity (0.52), connectivity density (0.52), and gray level nonuniformity (0.54); moderate negative correlation was found between osteoclast number and the Euler characteristic $(-0.56)(P<.01)$. Mild negative correlations between osteoclast number and high 
Table III. Summary of the bone perimeter, number of osteoclasts, and osteoclast density of each specimen

\begin{tabular}{lccc}
\hline Specimen & $\begin{array}{c}\text { Bone } \\
\text { Perimeter } \\
(\text { mm })\end{array}$ & $\begin{array}{c}\text { No. of } \\
\text { Osteoclasts }\end{array}$ & $\begin{array}{c}\text { Osteoclast Density } \\
\text { (no. of osteoclasts/mm) }\end{array}$ \\
\hline 1 L & 5.26 & 8 & 1.51 \\
2 L & 6.13 & 9.8 & 1.58 \\
2 R & 3.90 & 5.6 & 1.42 \\
3 L & 5.44 & 7.3 & 1.41 \\
3 R & 6.39 & 9.8 & 1.55 \\
4 L & 3.06 & 4.4 & 1.35 \\
$5 \mathrm{~L}$ & 3.59 & 0.7 & 0.21 \\
5 R & 3.82 & 0 & 0 \\
6 L & 4.50 & 4.9 & 1.11 \\
6 R & 3.02 & 3.1 & 1.03 \\
7 R & 4.48 & 3.8 & 0.89 \\
8 L & 3.65 & 4.8 & 1.35 \\
9 L & 4.96 & 4.3 & 0.87 \\
10 R & 3.39 & 5.5 & 1.37 \\
11 R & 4.73 & 2 & 0.43 \\
13 R & 5.04 & 10.6 & 2.08 \\
\hline
\end{tabular}

gray-level run emphasis $(-0.42)$ and short-run high graylevel emphasis $(-0.41)$ were observed $(P<.05)$. Similarly, mild negative correlations between osteoclast density and long-run high gray-level emphasis $(-0.39)$ and high graylevel run emphasis $(-0.43)$ were observed.

\section{DISCUSSION}

CBCT has been successfully used to characterize the osseous surface morphology of the maxillofacial region. ${ }^{23}$ Current CBCT machines can now resolve up to $80 \mu \mathrm{m},{ }^{24}$ which theoretically should be more than sufficient to image healthy human trabeculae that range in thickness from approximately 200 to $400 \mu \mathrm{m} .{ }^{25,26}$ However, few studies have been performed to determine if this is the case and if the CBCT machines used in dental practice today can, in fact, accurately characterize the internal structure of trabecular bone, for instance, in the mandibular condyle. Knowledge of such internal trabecular bone parameters may provide another aspect of bone morphology, which can aid in the diagnosis and monitoring of TMJ OA.

First, the results from our study show that the CBCT images had significantly higher measures for trabecular thickness, trabecular separation, bone volume, bone surface area and significantly lower measures for Euler characteristic, connectivity, and connectivity density compared with the micro-CT images. This was the expected result and can be attributed largely to the difference in voxel size between the CBCT and micro-CT images $(80 \mu \mathrm{m}$ vs $40 \mu \mathrm{m})$. Second, when trabecular bone is, in fact, detected within a voxel in a CBCT scan (even if, in reality, the trabecula does not completely fill the entire area of the voxel), a single gray value will be registered for the entire voxel. In comparison, this same area would comprise 8 different voxels in the higher resolu- tion micro-CT scan and thus would allow for varying gray-level values to be registered, depending on the amount of bone present in each of these 8 voxels. Thus, a larger voxel size not only omits a subset of the data that is below its threshold of detection but also creates a rounding error in them. Simply put, fine structures are not detected on CBCT, and thicker structures may appear even larger and dimensionally inaccurate. This is known as the partial volume averaging effect, which has been shown to contribute to erroneous bone dimensions and bone volume fractions in various medical imaging modalities. ${ }^{27,28}$ These findings were also qualitatively observed in the visual comparison of CBCT and microCT scans (Figure 1). Additionally, the image analysis procedures in the present study included multimodality registration to compare $\mathrm{CBCT}$ and micro-CT scans. Hill et al. ${ }^{29}$ described the registration of images taken with the same modality to assess longitudinal changes, and Behnami et al. ${ }^{30}$ discussed the use of multimodality registration to improve anatomic interpretation.

Although the absolute values for most of the bone parameters measured were significantly different between the CBCT and micro-CT scans, high Pearson correlations were observed for many bone structure parameters between the CBCT and micro-CT scans. Specifically, trabecular thickness, trabecular separation, bone volume, bone surface area, and degree of anisotropy showed strong significant correlations for the defect region. This indicates that these structural bone parameters may be utilized as potential bone imaging biomarkers in future $\mathrm{CBCT}$ studies of TMJ OA.

The only bone texture parameter that showed strong significant correlation between CBCT and micro-CT was the high gray-level run emphasis that may be used as potential imaging biomarker. All of the other gray-level runlength bone texture parameters showed either no correlation or a weak correlation, and thus, none of these parameters is considered a good candidate as a possible radiographic biomarker. Hounsfield units were not used to assess the gray level measurements in the present study. Parsa et al. ${ }^{31}$ and Pauwels et al. ${ }^{32}$ reported that gray values in CBCT scanners are higher than the Hounsfield units derived from MSCT because of increased noise, scattering, and artifacts in CBCT imaging. Pluim et al.$^{33}$ noted the challenges in using mutual information registration to measure the distribution of co-occurring gray intensity values in multimodality comparisons. The registration procedures in this study were not used for image subtraction or measurement of gray level differences. The registration in this study simply ensured that scans in both imaging modalities were assessed in similar orientations.

Our study also found osteoclasts localized in regions of subchondral defects in TMJ OA. This observation agrees with a previous study by Sabokbar et al. that found osteoclasts in subchondral cysts in cases of OA of the 

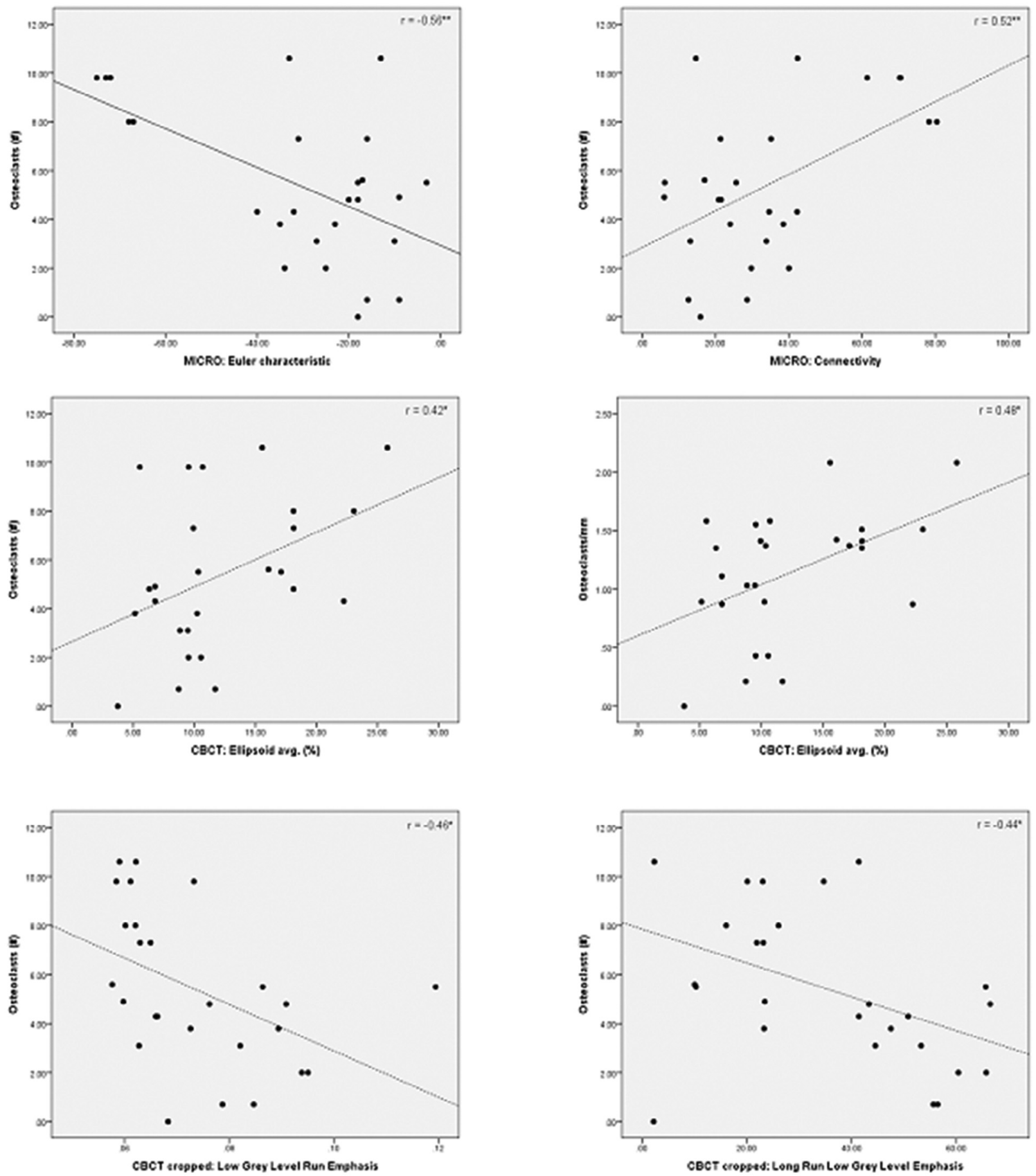

Fig. 4. Scatterplots showing correlations and linear regression between osteoclast number and density and the trabecular bone structure parameters from the cone beam computed tomography (CBCT) and micro-CT volumes for the corresponding specimens. Only statistically significant associations are illustrated. Insignificant associations were omitted. Note: $* P<.05, * * P<.01, * * * P<.001$.

femur. ${ }^{34}$ However, to the best of our knowledge, ours is the first study attempting to correlate osteoclast number and density with radiographic imaging parameters for TMJ $\mathrm{OA}$. An interesting finding of our study was the moderate correlations found between the number of osteoclasts in the histologic sections of the defective regions of the specimens and various bone structure parameters measured from the micro-CT volumes-namely, connectivity, connectivity density, and the Euler characteristic. These findings suggest that 3-D imaging can give us insight into 
Table IV. Pearson correlations of osteoclast number and density to 21 imaging parameters from cone beam computed tomography (CBCT) and micro-CT

\begin{tabular}{|c|c|c|c|c|}
\hline \multirow[b]{2}{*}{ Measurements $(n=26)$} & \multicolumn{2}{|c|}{ Correlation with $C B C T$} & \multicolumn{2}{|c|}{ Correlation with Micro-CT } \\
\hline & Osteoclast No. & Osteoclast No. / mm & Osteoclast No. & Osteoclast No. / mm \\
\hline Trabecular bone structure parameters & -0.34 & -0.38 & -0.36 & -0.36 \\
\hline Trabecular thickness (mean) (mm) & 0.30 & 0.34 & -0.17 & -0.01 \\
\hline Trabecular separation (mean) (mm) & -0.34 & -0.35 & -0.08 & -0.12 \\
\hline Bone volume/total volume & -0.28 & -0.32 & 0.14 & 0.07 \\
\hline Bone surface area $\left(\mathrm{mm}^{2}\right)$ & 0.03 & 0.02 & -0.29 & -0.28 \\
\hline Structural model index & 0.26 & 0.33 & 0.00 & 0.08 \\
\hline Degree of anisotropy & 0.23 & 0.28 & $-0.56^{*}$ & -0.33 \\
\hline Euler characteristic & -0.14 & -0.18 & $0.52^{*}$ & 0.31 \\
\hline Connectivity & -0.14 & -0.18 & $0.52 *$ & 0.31 \\
\hline Connectivity density $\left(/ \mathrm{mm}^{3}\right)$ & $0.42^{\dagger}$ & $0.48^{\dagger}$ & -0.32 & -0.34 \\
\hline \multicolumn{5}{|l|}{ Ellipsoid factor $(\%)$} \\
\hline \multicolumn{5}{|l|}{ Grey level run-length } \\
\hline \multicolumn{5}{|l|}{ Bone texture parameters } \\
\hline Short run emphasis & -0.16 & -0.11 & 0.06 & 0.19 \\
\hline Long run emphasis & 0.19 & 0.16 & -0.19 & -0.33 \\
\hline Gray-level nonuniformity & -0.07 & -0.05 & $0.54^{*}$ & 0.35 \\
\hline Run length nonuniformity & -0.36 & -0.23 & 0.27 & 0.18 \\
\hline Low gray-level run emphasis & $-0.46^{\dagger}$ & -0.36 & 0.35 & 0.32 \\
\hline High gray-level run emphasis & 0.16 & 0.13 & $-0.42^{\dagger}$ & $-0.39^{\dagger}$ \\
\hline Short run low gray-level emphasis & -0.21 & -0.13 & 0.30 & 0.33 \\
\hline Short run high gray-level emphasis & -0.001 & -0.02 & $-0.41^{\dagger}$ & -0.31 \\
\hline Long run low gray-level emphasis & $-0.44^{\dagger}$ & -0.37 & 0.08 & -0.05 \\
\hline Long run high gray-level emphasis & 0.32 & 0.31 & -0.33 & $-0.43^{\dagger}$ \\
\hline
\end{tabular}

the degenerative cellular processes occurring within the condyle. For instance, when parameters are increased, degenerative metabolic processes that upregulate osteoclasts may be at play. However, it is difficult to directly draw this conclusion as confounding factors may have contributed to the results, and micro-CT measurements cannot be clinically applied because of their inherent limitations. For example, connectivity is an approximation of the number of trabeculae in the sample, and connectivity density gives an approximation of the density of trabeculae (i.e., trabeculae per $\mathrm{mm}^{3}$ ), whereas the Euler characteristic is a mathematical representation of the number of connected structures in a network. As can be seen, all these measures are themselves correlated to the number of trabeculae. Therefore, it may simply be that in a sample with more trabeculae, there is a greater likelihood of detecting osteoclasts because there are proportionally more regions in which they can be localized. It is also important to note that these 3 bone structure parameters only showed significant correlations to the raw number of osteoclasts per slide but not to osteoclast density. Thus, the correlations observed may be more of a result of the parameter being measured rather than an underlying biologic process. Further, it was also found that osteoclast number and density were significantly correlated with ellipsoid factor measured from $\mathrm{CBCT}$ volumes; however, ellipsoid factor from the micro-CT volumes, the gold standard, was not significantly correlated with either osteoclast number or density, and thus, this is likely an aberrant finding.

Only 2 other studies reported in the literature have investigated the accuracy of CBCT compared with micro$\mathrm{CT}$ in imaging mandibular structures. ${ }^{35,36}$ Each of these studies compared CBCT and micro-CT in measuring a different set of trabecular bone parameters, and our results generally agree with the findings in these studies. Ibrahim et al. ${ }^{35}$ demonstrated a similar Pearson correlation between micro-CT and CBCT for the measurement of trabecular thickness in the posterior mandible of human cadavers $(r=0.82)$ and also showed a significant correlation for trabecular separation $(r=0.94)$ between the 2 imaging modalities. Ho et al. ${ }^{36}$ demonstrated that $\mathrm{CBCT}$ and microCT measurements of trabecular thickness, trabecular separation, bone surface area, and bone volume fraction were all highly correlated with Spearman correlations ranging from 0.8 to 0.96 . However, a significant difference between our study and Ho et al.'s study was that Ho et al. imaged uniform synthetic bone blocks made from polyurethane plastic, and this was a limitation of that study.

The results of our study were quite encouraging in that the trabecular bone structural parameters measured from volumes acquired on a CBCT machine commonly used in the dental setting are representative of the true nature of bone. Specifically, trabecular thickness, trabecular sep- 
aration, bone volume, bone surface area and degree of anisotropy are structural bone parameters that show promise to be utilized as potential bone imaging biomarkers in future CBCT studies of TMJ OA. It is foreseeable that as the CBCT technology continues to evolve, trabecular characterization will be utilized for screening, diagnosis, and monitoring of TMJ OA.

\section{CONCLUSIONS}

In our study, we observed strong correlations among the trabecular thickness, trabecular separation, bone volume, bone volume/total volume, bone surface area, and degree of anisotropy measurements in both CBCT and micro$\mathrm{CT}$ images. This indicates that these parameters may be suitable bone imaging biomarkers that can be utilized clinically.

Moderate correlations were found between osteoclast number and 3 trabecular bone structure measurements-connectivity, connectivity density, and the Euler characteristic. However, these 3 bone structure parameters did not significantly correlate with osteoclast density. Thus, this conclusion should be interpreted with caution because of the several confounding factors that could have led to this result.

The authors would like to acknowledge James Sugai, William Giannobile, and Chris Strayhorn, who provided technical help and general assistance with the histologic evaluations.

\section{REFERENCES}

1. Alomar X, Medrano J, Cabratosa J, et al. Anatomy of the temporomandibular joint. Semin Ultrasound CT MR. 2007;28:170183.

2. Sokoloff L. The biology of degenerative joint disease. Perspect Biol Med. 1963;7:94-106.

3. Buckwalter JA, Mankin HJ. Articular cartilage: degeneration and osteoarthritis, repair, regeneration, and transplantation. Instr Course Lect. 1998;47:487-504.

4. Tanaka E, Detamore MS, Mercuri LG. Degenerative disorders of the temporomandibular joint: etiology, diagnosis, and treatment. J Dent Res. 2008;87:296-307.

5. Mejersjo C. Therapeutic and prognostic considerations in TMJ osteoarthrosis: a literature review and a long-term study in 11 subjects. Cranio. 1987;5:69-78.

6. Kalladka M, Quek S, Heir G, Eliav E, Mupparapu M, Viswanath A. Temporomandibular joint osteoarthritis: diagnosis and longterm conservative management: a topic review. J Indian Prosthodont Soc. 2014;14:6-15.

7. Rasmussen OC. Temporomandibular arthropathy. Clinical, radiologic, and therapeutic aspects, with emphasis on diagnosis. Int $J$ Oral Surg. 1983;12:365-397.

8. Mercuri LG. Surgical management of TMJ arthritis. In: Laskin D.M., Greene C.S., Hylander W.L., eds. Temporomandibular Disorders: An Evidence-Based Approach to Diagnosis and Treatment. Chicago, IL: Quintessence; 2006:455-468.

9. de Souza RF, Lovato da Silva CH, Nasser M, Fedorowicz Z, AlMuharraqi MA. Interventions for the management of temporomandibular joint osteoarthritis. Cochrane Database Syst Rev. 2012;(4):CD007261.
10. Manzione JV, Katzberg RW, Brodsky GL, Seltzer SE, Mellins HZ. Internal derangements of the temporomandibular joint: diagnosis by direct sagittal computed tomography. Radiology. 1984;150: 111-115.

11. Honey OB, Scarfe WC, Hilgers MJ, et al. Accuracy of conebeam computed tomography imaging of the temporomandibular joint: comparisons with panoramic radiology and linear tomography. Am J Orthod Dentofacial Orthop. 2007;132:429-438.

12. Peltonen LI, Aarnisalo AA, Kortesniemi MK, Suomalainen A, Jero J, Robinson S. Limited cone-beam computed tomography imaging of the middle ear: a comparison with multislice helical computed tomography. Acta Radiol. 2007;48:207-212.

13. Burr DB. The importance of subchondral bone in osteoarthrosis. Curr Opin Rheumatol. 1998;10:256-262.

14. Brandt KD, Schauwecker DS, Dansereau S, Meyer J, O'Connor B, Myers SL. Bone scintigraphy in the canine cruciate deficiency model of osteoarthritis. Comparison of the unstable and contralateral knee. J Rheumatol. 1997;24:140-145.

15. Fazzalari NL, Parkinson IH. Fractal properties of subchondral cancellous bone in severe osteoarthritis of the hip. J Bone Miner Res. 1997;12:632-640.

16. Chappard D, Guggenbuhl P, Legrand E, Basle MF, Audran M. Texture analysis of $\mathrm{X}$-ray radiographs is correlated with bone histomorphometry. J Bone Miner Metab. 2005;23:24-29.

17. Chappard D, Basle MF, Legrand E, Audran M. Trabecular bone microarchitecture: a review. Morphologie. 2008;92:162-170.

18. Galloway MM. Texture analysis using gray level run lengths. Comput Graph Image Process. 1975;4:172-179.

19. Dworkin SF, LeResche L. Research diagnostic criteria for temporomandibular disorders: review, criteria, examinations and specifications, critique. J Craniomandib Disord. 1992;6:301-355.

20. Ahmad M, Hollender L, Anderson Q, et al. Research diagnostic criteria for temporomandibular disorders (RDC/TMD): development of image analysis criteria and examiner reliability for image analysis. Oral Surg Oral Med Oral Pathol Oral Radiol Endod. 2009;107:844-860.

21. Doube M, Klosowski MM, Arganda-Carreras I, et al. BoneJ: free and extensible bone image analysis in ImageJ. Bone. 2010;47: 1076-1079.

22. Ross MH, Pawlina W. Histology - A Text and Atlas with Correlated Cell and Molecular Biology. 5th ed. Baltimore, MD: Lippincott Williams \& Wilkins; 2006.

23. De Vos W, Casselman J, Swennen GR. Cone-beam computerized tomography (CBCT) imaging of the oral and maxillofacial region: a systematic review of the literature. Int J Oral Maxillofac Surg. 2009;38:609-625.

24. Gonzalez-Garcia R, Monje F. The reliability of cone-beam computed tomography to assess bone density at dental implant recipient sites: a histomorphometric analysis by micro-CT. Clin Oral Implants Res. 2013;24:871-879.

25. Fanuscu MI, Chang TL. Three-dimensional morphometric analysis of human cadaver bone: microstructural data from maxilla and mandible. Clin Oral Implants Res. 2004;15:213-218.

26. Moon HS, Won YY, Kim KD, et al. The three-dimensional microstructure of the trabecular bone in the mandible. Surg Radiol Anat. 2004;26:466-473.

27. Adams JE. Quantitative computed tomography. Eur J Radiol. 2009; $71: 415-424$.

28. Hoffman EJ, Huang SC, Phelps ME. Quantitation in positron emission computed tomography: 1. Effect of object size. J Comput Assist Tomogr. 1979;3:299-308.

29. Hill DL, Batchelor PG, Holden M, Hawkes DJ. Medical image registration. Phys Med Biol. 2001;46:R1-R45.

30. Behnami D, Seitel A, Rasoulian A, et al. Joint registration of ultrasound, CT and a shape+pose statistical model of the lumbar spine 
for guiding anesthesia. Int J Comput Assist Radiol Surg. 2016; 11:937-945

31. Parsa A, Ibrahim N, Hassan B, Motroni A, van der Stelt P, Wismeijer D. Influence of cone beam CT scanning parameters on grey value measurements at an implant site. Dentomaxillofac Radiol. 2013;42:79884780.

32. Pauwels R, Jacobs R, Singer SR, Mupparapu M. CBCT-based bone quality assessment: are Hounsfield units applicable? Dentomaxillofac Radiol. 2015;44:20140238.

33. Pluim JP, Maintz JB, Viergever MA. Mutual-information-based registration of medical images: a survey. IEEE Trans Med Imaging. 2003;22:986-1004.

34. Sabokbar A, Crawford R, Murray DW, Athanasou NA. Macrophage-osteoclast differentiation and bone resorption in osteoarthrotic subchondral acetabular cysts. Acta Orthop Scand. 2000;71:255-261.

35. Ibrahim N, Parsa A, Hassan B, van der Stelt P, Aartman IH, Wismeijer D. Accuracy of trabecular bone microstructural measurement at planned dental implant sites using cone-beam CT datasets. Clin Oral Implants Res. 2014;25:941-945.

36. Ho JT, Wu J, Huang HL, Chen MY, Fuh LJ, Hsu JT. Trabecular bone structural parameters evaluated using dental cone-beam computed tomography: cellular synthetic bones. Biomed Eng Online. 2013;12:115

\section{Reprint requests:}

Antonio C.O. Ruellas, PhD

Department of Pediatric Dentistry and Orthodontics Federal University of Rio de Janeiro

Rua Prof Rodolpho Paulo Rocco, 325, 21941-617

Rio de Janeiro

Brazil

antonioruellas@yahoo.com.br 